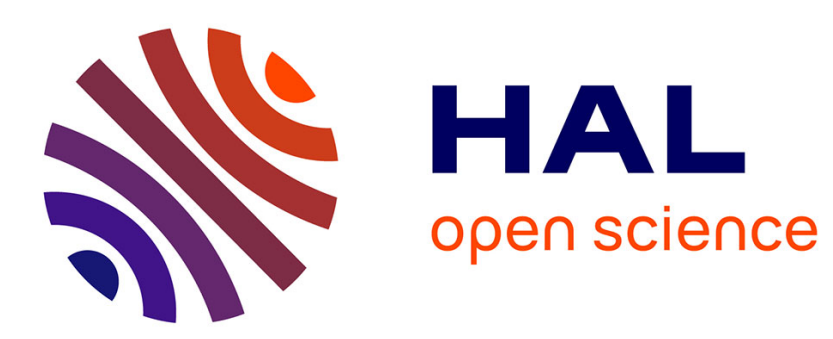

\title{
Contextual Multi-Scale Image Classification on Quadtree
} Ihsen Hedhli, Gabriele Moser, Sebastiano B. Serpico, Josiane Zerubia

\section{To cite this version:}

Ihsen Hedhli, Gabriele Moser, Sebastiano B. Serpico, Josiane Zerubia. Contextual Multi-Scale Image Classification on Quadtree. IEEE International Conference on Image Processing (ICIP), Sep 2016, Phoenix, United States. hal-01316611

\section{HAL Id: hal-01316611 \\ https://hal.inria.fr/hal-01316611}

Submitted on 17 May 2016

HAL is a multi-disciplinary open access archive for the deposit and dissemination of scientific research documents, whether they are published or not. The documents may come from teaching and research institutions in France or abroad, or from public or private research centers.
L'archive ouverte pluridisciplinaire HAL, est destinée au dépôt et à la diffusion de documents scientifiques de niveau recherche, publiés ou non, émanant des établissements d'enseignement et de recherche français ou étrangers, des laboratoires publics ou privés. 
CONTEXTUAL MULTI-SCALE IMAGE CLASSIFICATION ON QUADTREE Ihsen Hedhli ${ }^{* \dagger}$, Gabriele Moser ${ }^{\dagger}$, Sebastiano B. Serpico ${ }^{\dagger}$ and Josiane Zerubia ${ }^{*}$

\author{
*INRIA, Sophia-Antipolis Méditerranée Center, AYIN team, France, firstname.lastname@inria.fr \\ ${ }^{\dagger}$ DITEN, Department of Electrical, Electronic, and Telecommunications Engineering \\ and Naval Architecture - University of Genoa, Italy, firstname.lastname@ unige.it
}

\begin{abstract}
In this paper, we propose a novel hierarchical method for remote sensing image classification. The proposed approach integrates an explicit hierarchical graph-based classifier, which uses a quad-tree structure to model multiscale interactions, and a third order Markov mesh random field to deal with pixel wise contextual information in the same scale. The choice of a quad-tree and the third order Markov mesh allow taking benefit from their good analytical properties (especially causality) and consequently apply non-iterative algorithms. Indeed, the Markov mesh is used to incorporate spatial information in each scale of the quad-tree while keeping the causality of the hierarchical model.
\end{abstract}

Index Terms - Multi-scale classification, causal model, hierarchical MRF, Markov mesh, MPM.

\section{INTRODUCTION}

Nowadays, a substantial amount and variety of lastgeneration high resolution (HR) satellite missions provide images acquired simultaneously at different spatial and spectral resolutions. The main difficulty is to develop a classifier that jointly utilizes the benefits of multi-band and multi-resolution input data while maintaining a good tradeoff between accuracy and computation time. Classification technique as an example of inverse problems can be regarded as the process that estimates hidden information (or latent variables) $x$ (i.e., land cover class labels) from observations $y$ (i.e., satellite data) attached to a set of nodes S. Hence, this problem is ill posed in the sense of Hadamard [1]. The classical way to handle this disadvantage is to regularize the solution by imposing prior knowledge on the labels, which may include, most remarkably, spatial-contextual priors. In this framework, Markov random field (MRF) models are widely used in image classification since they provide a convenient and consistent way of integrating contextual information into the classification scheme [2,5]. Because of their generally non-causal nature, MRF models for classification lead to iterative inference algorithms that are computationally demanding [6-10]. By contrast, MRF models defined according to hierarchical structures exhibit good methodological and application-oriented properties including causality by using appropriate graphs [11]. Indeed, from a random process perspective, Markovian models show a strong bonding with graphs [4]. One of the advantage of using graphs in a probabilistic framework is the possibility of immediate visual interpretation of the relationships between variables that expresses different dependency among the nodes. In fact, the graphical viewpoint allows in some cases to identify at first glance interaction structures that always support causal models allowing the use of non-iterative algorithms with acceptable computational time [12].

The aim of the present paper is to develop a multi-resolution classifier using remote sensing data. The input images are inserted in a hierarchical structure on the basis of their spatial resolutions. This approach is aimed at both exploiting multiscale information, which is known to play a crucial role in high-resolution image analysis, and supporting contextual information at each scale.

To deal with the hierarchical aspect, several causal methods have been developed using quad-tree structures [11-17]. In practice, this structure sometimes yields "blocky" effects in the final classification map. Here, the quad-tree approach is extended and a novel contextual multi-scale technique is proposed in order to classify multi-resolution remote sensing data that incorporate spatial contextual information and mitigate possible blocky artifacts. Indeed, Markov mesh model [18] is used to incorporate spatial information in each scale of the quad-tree while keeping the causality of the hierarchical model.

\section{METHODOLOGY}

\subsection{Causal hierarchical image models}

Using graphical representations, probabilistic causal image models have been thoroughly studied since the early 80 's [1114]. The classes of causal autoregressive fields [12], unilateral MRFs [18], mesh MRFs [18], and hierarchical MRFs on quad-trees $[12,14,15]$ have thus been introduced. These models rely on a causality concept captured by the factorization of the prior distribution in terms of causal transition probabilities [19].

This concept implicitly requires an order over the set of sites $S$, i.e., a well-defined characterization of the "past" of a site $\mathrm{s}$, as a set of nodes preceding $s$. Given the ordered set $S=$ $\{1, \ldots s-1, s, s+1, \ldots, N\}$ the past of the site $s$ will be defined by $p a(s)=\{i \in S: i<s\}$. Thus, the causality is used 
to define a subset of site $\partial_{s} \subset p a(s)$ that seeks the following property:

$$
\forall s>1, p\left(x_{s} \mid x_{p a(s)}\right)=p\left(x_{s} \mid x_{\partial_{s}}\right),
$$

If (1) holds, one can break the joint distribution $p(x)$ $\left(x=\left(x_{1}, \ldots, x_{N}\right)\right)$ as a product of the conditional probabilities over the past neighborhood. Formally:

$$
\begin{aligned}
p(x)=p\left(x_{1}\right) \prod_{s=2}^{N} p\left(x_{s} \mid x_{p a(s)}\right) & \\
& =p\left(x_{1}\right) \prod_{s=2}^{N} p\left(x_{s} \mid x_{\partial_{s}}\right),
\end{aligned}
$$

The most remarkable point about (2) is the absence of a normalizing constant, which results in performing efficient and non-iterative techniques.

In the case of a Markovian process using a neighborhood system $\aleph=\left\{\vartheta_{s}, s \in S\right\}$, where $\vartheta_{s}$ is the local neighborhood of $s, \partial_{s}$ is defined as:

$$
\forall s \in S, \quad \partial_{s} \subseteq \vartheta_{s} \cap p a(s)
$$

It has been shown in [19] that graphs satisfying the characterization in (3) with respect to an ordered set $S$ are triangulated (or chordal), i.e., they contain no cycles of length 4 without a chord.

In the case of causal MRFs, on a lattice also called Markov mesh random fields (MMRFs, [18, 20-22]), the predecessors of the pixel $s=(i, j)$ are shown in shaded cells in Figure 1 and the Markovian neighborhood if $s$ is in blue. The resulting causal neighborhood is the intersection of these two sets. The "order" of a MMRF corresponds of the number of "causal" neighbors for each site. (a) Second order MMRF

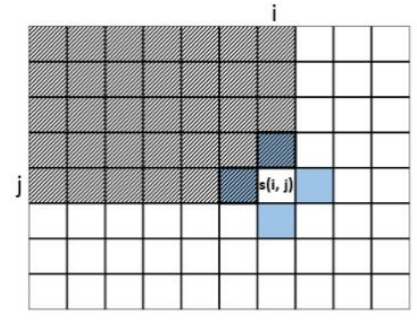

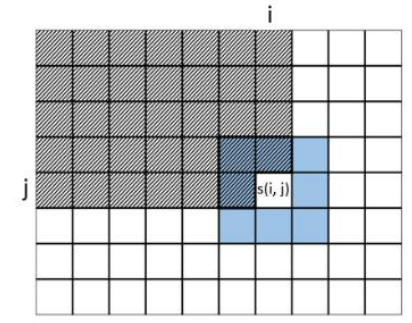

(b) Third order MMRF
Figure 1: Causal neighborhood

The main objective of this paper is to develop a method for multi-resolution classification based on a causal hierarchical Markovian model. As shown in Figure 2(b), pyramidal schemes are one of the possible structures in which images are organized according to their resolutions. Then, to simplify the interactions between different images in the pyramid, we define for each node of the pyramid a set of links to other nodes to model scale-to-scale interactions. The theory of multiscale signals has been widely studied, and their representations lead naturally to models of signals on trees [23, 24]. Among others, quad-trees have been proposed as attractive candidates for modeling these scale-to-scale interactions. As recalled in the previous section, the selection of these structures is justified by their causality properties over scale as they are triangulated by definition for not having cycles.

In the quad-tree, let us define an upward shift operator $\delta$ such that $s^{-}=\delta(s)$ is the parent of node $s$. Note that the operator $\delta$ is four-to-one since each parent has four offsprings. Also, we define the forward shift operator $\beta$ such that $s^{+}=\beta(s)$ is a descendant of $s$, the interchange operator $\alpha$ between nodes in the same scale, and $d(s)$ is the set including $s$ and all its descendants in the tree, as illustrated in Figure 2(a)

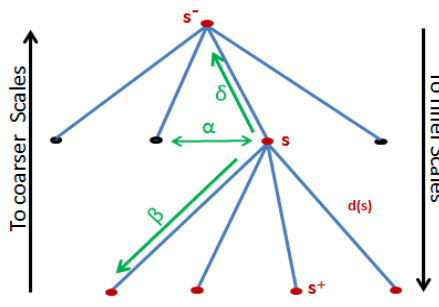

(a) Quad tree structure

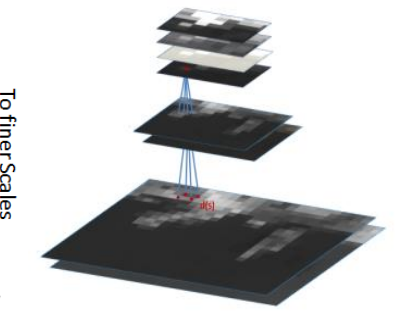

(b) Pyramidal structure
Figure 2: Hierarchical structure

\subsection{The proposed model}

The aim of the classification is to estimate the set of latent variables $\chi$ (here, land cover class labels) given a set of observations $\Upsilon$ (here, satellite data) attached to the set of nodes (pixels) $\mathrm{S}$. Each label takes a value in the set $\Lambda=$ $\{0,1, \ldots, M-1\}$ of possible classes. The configuration space $\Omega=\Lambda^{|S|}$ is the set of all global discrete labelings. $\chi=$ $\left\{x_{s}\right\}_{s \in S}, x_{s} \in \Lambda$ and $\Upsilon=\left\{\mathrm{y}_{\mathrm{s}}\right\}_{\mathrm{s} \in \mathrm{S}}$ are recursively the random fields of the class labels and observations of all nodes. In this context, we consider the problem of inferring the "best" configuration $\hat{x} \in \Omega$.

When the causality property holds, non-iterative classification algorithms can be applied. In particular, a recursive procedure on a quad-tree is feasible for the marginal posterior modes (MPM) criterion [14], in which the aim is to maximize the posterior marginal at each site s:

$$
\forall s \in S, \hat{x}_{s}=\arg \max _{x_{S} \in \Lambda} p\left(x_{s} \mid y\right),
$$

which produces the configuration that maximizes at each site $s$ the a posteriori marginal $p\left(x_{s} \mid y\right)$.

Because the tree is acyclic, the labels are estimated recursively through a forward-backward algorithm similar to the classical Baum and Welch technique for Markov chains [25]. In practice, the use of one quad-tree structure with the MPM criterion often yields "blocky" effects [14]. This phenomenon can be explained by the fact that two neighboring sites at a given scale may not have the same parent.

In this paper, a novel hybrid structure that combines a spatial grid using a causal MMRF and a hierarchical MRF via quadtree is employed to circumvents the blocky artifacts of quadtree-based models and incorporates spatial contextual information in each scale. 
The starting point is to give an order on the set of all nodes $S$. For each scale of the quad-tree, a causal third-order MMRF is integrated into the hierarchical structure. Accordingly, a node $s$ is depend on one parent and three neighbors for each scale of the quad-tree (except for pixels in the top of the pyramid where there is no a parent node and pixels in the bottom and left borders of each lattice at each scale of the pyramid where there is no MMRF neighborhood) as illustrated in the dependence graph in figure 3.

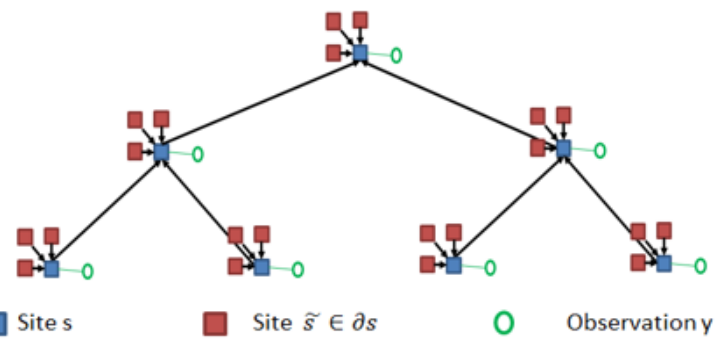

Figure 3: Independence graph of the joint model $(\chi, Y)$

To resolve recursively the optimization problem in (4), the posterior marginal $\mathrm{p}\left(\mathrm{x}_{\mathrm{s}} \mid \mathrm{y}\right)$ of the label of each node $\mathrm{s}$ is expressed as a function of the posterior marginal $\mathrm{p}\left(\mathrm{x}_{\mathrm{s}}-\mid \mathrm{y}\right)$ of the parent node $s^{-}$in the quad-tree and the posterior marginals $\mathrm{p}\left(\mathrm{x}_{\tilde{\mathrm{s}}} \mid \mathrm{y}\right)$ where $\tilde{\mathrm{s}} \in \partial \mathrm{s}$ and $\partial \mathrm{s}$ is the set of the three causal neighbors of s. Formally:

$$
\begin{aligned}
& \boldsymbol{p}\left(\boldsymbol{x}_{\boldsymbol{s}} \mid \boldsymbol{y}\right) \\
& =\sum_{x_{s}^{-}, x_{\partial s}} \frac{p\left(x_{s}, x_{s^{-}}, x_{\partial s} \mid y_{d(s)}\right)}{\sum_{x_{s}} p\left(x_{s}, x_{s^{-}}, x_{\partial s} \mid y_{d(s)}\right)} \boldsymbol{p}\left(\boldsymbol{x}_{\boldsymbol{s}^{-}} \mid \boldsymbol{y}\right) \prod_{\tilde{\mathbf{s}} \in \partial s} \boldsymbol{p}\left(\boldsymbol{x}_{\tilde{\mathbf{s}}} \mid \boldsymbol{y}\right)
\end{aligned}
$$

where bold denotes the marginal posteriors of interest. For analytical convenience, equation (5) involves two conditional independence assumptions: (i) the distribution of the labels $\left(x_{s}, x_{s^{-}}, x_{\partial s}\right)$ given all the observations $y$ can be restricted to the distribution conditioned only to the descendants of site $s$; and (ii) the labels $\widetilde{\mathrm{s}}$ of the three causal neighbors $\partial \mathrm{s}$ are independent when conditioned to the data $y$. This formulation allows calculating recursively the posterior marginal $p\left(x_{s} \mid y\right)$ at each site $s$ while the probabilities $p\left(x_{s}, x_{s^{-}}, x_{\partial s} \mid y_{d(s)}\right)$ are made available. Thus, under the conditional independence assumption that, the distribution of the labels $s^{-}$and $\partial s$ are independent on the observations $y_{d(s)}$, when conditioned to the label $x_{s}$ the computation of these joint probabilities boils down to the determination of the other probabilities involved in (6):

$$
\begin{aligned}
& p\left(x_{s}, x_{s^{-}}, x_{\partial s} \mid y_{d(s)}\right) \\
& =\frac{p\left(x_{s} \mid x_{s^{-}}\right) \cdot p\left(x_{s^{-}}\right)}{p\left(x_{s}\right)} \cdot \prod_{\tilde{s} \in \partial \mathrm{s}} \frac{p\left(x_{s} \mid x_{\tilde{s}}\right) \cdot p\left(x_{\tilde{s}}\right)}{p\left(x_{s}\right)} \cdot p\left(x_{s} \mid y_{d(s)}\right)
\end{aligned}
$$

where the factor $p\left(x_{s} \mid x_{s^{-}}\right)$corresponds to the childparent transition probability; $p\left(x_{s}\right)$ is the prior probability; $p\left(x_{s} \mid x_{\tilde{\mathrm{s}}}\right)$ is the transition probability at the same scale and $p\left(x_{s} \mid y_{d(s)}\right)$ is the partial posterior marginal probability.

To maximize the posterior marginal at each pixel, we take benefit from the hierarchical structure defined above and we use two recursive passes on the quad-tree, referred to as "bottom-up" and "top-down" passes as shown in Algorithm1.

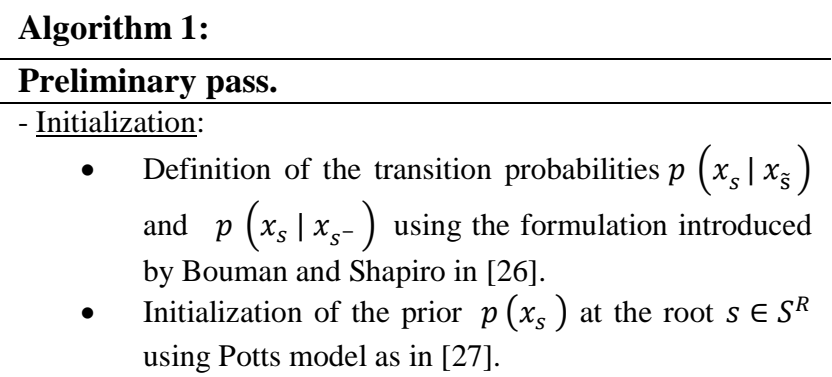

$$
\begin{aligned}
& \text { - Recursion: } s \in\left\{S^{R-1}, \ldots, S^{0}\right\} . \\
& \bullet \quad p\left(x_{s}\right)=\sum_{x_{s^{-}}} p\left(x_{s} \mid x_{s^{-}}\right) \cdot p\left(x_{s^{-}}\right)
\end{aligned}
$$

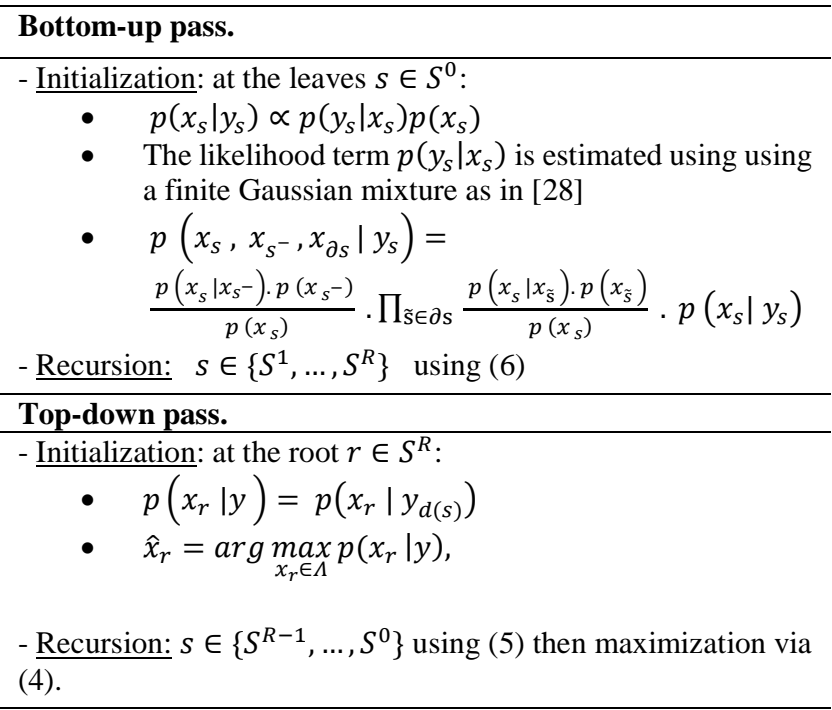

\section{EXPERIMENTAL RESULTS}

In this section, we discuss the results of the experimental validation of the developed contextual hierarchical classifier using panchromatic and multispectral Pléiades images acquired over Port-au-Prince (Haiti). The finest resolution of the multiresolution pyramid (level 0) was set equal to the finest resolution of the input panchromatic images (i.e., 0.5 $\mathrm{m}$ ). Co-registered multispectral images (at $2 \mathrm{~m}$ ) were integrated in level 2 of the pyramid. To fill level 1, a wavelet decomposition of the panchromatic image was used.

Five land cover classes have been considered: urban (red), water (blue), vegetation (green), soil (yellow) and containers 
(purple). In the present work, manually annotated (given by an expert) non-overlapping training and test sets were selected in homogeneous areas. Spatially disjoint training and test areas were used. The accuracies reported in Table 1 are obtained by comparing classification results to manually annotated test sets. The proposed approach formalizes a supervised Bayesian classifier within quad-tree topology that combines a class conditional statistical model for pixel-wise information and a hierarchical MRF for multi-resolution contextual information. Given a training set for each input data, for each class $m$ and scale $n$, we model the corresponding class-conditional marginal using finite mixtures of independent gray level distributions. The mixture modeling is performed depending on the type of remote sensing imagery used in the study. Indeed, when the input data at the $n^{\text {th }}$ scale level is an optical image, classconditional marginal PDF related to each class $m$ can be modeled by a Gaussian mixture model. As shown in Figures 4, 5 and table 1 , the resulting classification map shows that the proposed hierarchical method leads to accurate results, especially as compared to the original hierarchical classification technique based on the MPM criterion in [14] which yields to "blocky" classification (see Figures 1(c) and 2 (c)). These blocky artifacts are mitigated by incorporating spatial contextual information. The proposed method was also compared to an extended version of Laferté et al. [14] method in which the blocky artifacts were reduced by employing a prior update technique in the top-down step of the hierarchical algorithm as described in [15]. Comparing to this method, the proposed technique obtains higher accuracies especially over urban area as shown in Figures 4, 5 and Table 1.

\section{CONCLUSIONS}

The proposed method is aimed at performing hierarchical classification using input multiresolution imagery. It combines a causal hierarchical MRF model using a quad-tree and a Markov mesh to preserve contextual information at each scale by applying a non-iterative classification algorithm using the MPM criterion. The paper is primarily focused on the classification of multiresolution remote sensing images. The proposed model is nonetheless general, and could be applied to other imaging contexts, for instance medical imaging. Experimental results with VHR satellite imagery show that the method allows to effectively incorporate spatial information in the hierarchical classification process and provides higher accuracies than previous benchmark techniques.
A major advantage of the proposed classifier is that it can be extended to the use of synthetic aperture radar (e.g. COSMOSkyMed, TerraSAR-X, RADARSAR-2) or multisensor data. The extension to the multisensor case will be a major direction of future research.

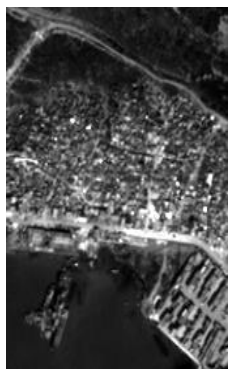

(a)

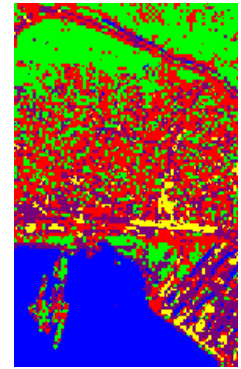

(c)

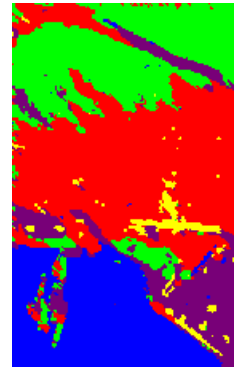

(d)

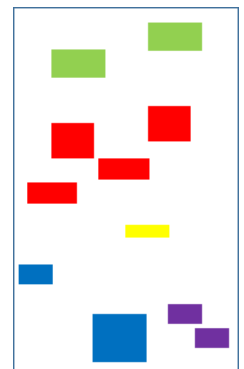

(b)
Figure 4: classification maps of image (a) using the original method of Laferté et al. (c), the proposed method (d) and Voisin et al. method (e) and using the ground truth (b).

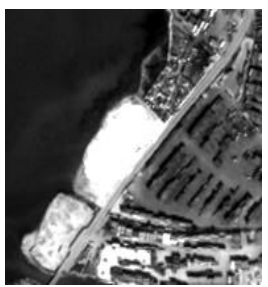

(a)

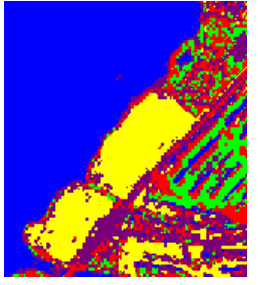

(c)

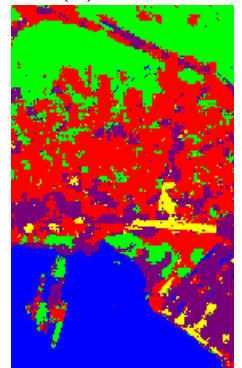

(e)

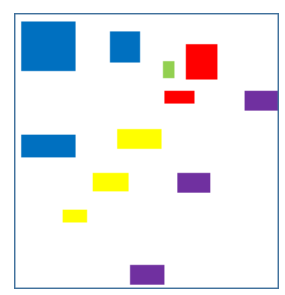

(b)

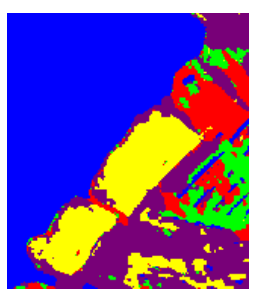

(d)

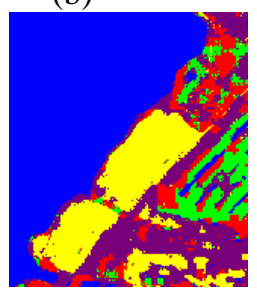

(e)
Fure 5: classification maps of image (a) using the original method of Laferté et al. (c), the proposed method (d), and Voisin et al. method (e) and using the ground truth (b).

\begin{tabular}{|l|c|c|c|c|c|c|c|}
\cline { 2 - 8 } \multicolumn{1}{c|}{} & water & urban & vegetation & containers & soil & Over all & Computation time \\
\hline Method in [14] & $100 \%$ & $62 \%$ & $76 \%$ & $72 \%$ & $91 \%$ & $80.2 \%$ & 120 seconds \\
\hline Method in [15] & $100 \%$ & $74 \%$ & $83 \%$ & $\mathbf{8 6 \%}$ & $92 \%$ & $87 \%$ & 154 seconds \\
\hline The proposed method & $\mathbf{1 0 0 \%}$ & $\mathbf{9 2 \%}$ & $\mathbf{8 9 \%}$ & $81 \%$ & $\mathbf{9 4 \%}$ & $\mathbf{9 1 . 2 \%}$ & 147 seconds \\
\hline
\end{tabular}

Table 1: Results obtained using the Pléiades dataset: class accuracies, overall accuracy, and computation time. Experiments were conducted on an Intel i7 quad-core $(2.40 \mathrm{GHz})$ 8-GB-RAM 64-bit Linux system. 


\section{REFERENCES}

[1] J. Hadamard. Lectures on Cauchy's problem in linear partial differential equations, Courier Corporation, 1923.

[2] P. Fieguth, Statistical Image Processing and Multidimensional Modeling, Springer, New York, 2011.

[3] Z. Kato and J. Zerubia. Markov Random Fields in Image Segmentation, NOW publishers, Boston, 2012.

[4] S.Z. Li, Markov Random Field Modeling in Image Analysis, Springer, 3rd Edition, New York, 2009.

[5] G. Winkler, Image Analysis, Random Fields and Markov Chain Monte Carlo Methods, Springer, New York, 2003.

[6] S. Geman and D. Geman, "Stochastic relaxation, Gibbs distributions, and the Bayesian restoration of images," IEEE Trans. Pattern Anal. Machine Intel, vol. PAMI-6, pp. 721-741, 1984.

[7] P. Brémaud, Markov chains: Gibbs fields, Monte Carlo simulation, and queues, Springer, New York, 2013.

[8] S. Rajasekaran, "On the convergence time of simulated annealing," Research Report MS-CIS-90-89, University of Pennsylvania, Department of Computer and Information Science, USA, November 1990.

[9] J. Besag, "On the statistical analysis of dirty pictures." Journal of the Royal Statistical Society. Series B (Methodological), 259302, 1986.

[10] Y. Boykov, O. Veksler, R. Zabih, "Fast approximate energy minimization via graph cuts", IEEE Transactions on Pattern Analysis and Machine Intelligence, vol.23, no.11, pp.1222-1239, 2001.

[11] A.S. Willsky, "Multiresolution Markov models for signal and image processing." Proceedings of the IEEE 396-1458, 2002.

[12] M. Luettgen, W. Karl and A. Willsky. "Efficient multiscale regularization with applications to the computation of optical flow", IEEE Trans. Image Processing, vol. 3, 41-64, 1994.

[13] Patrick Pérez, Annabelle Chardin, and Jean-Marc Laferté. Noniterative manipulation of discrete energy-based models for image analysis. Pattern Recognition, 33 (4):573-586, 2000.

[14] J.M. Laferté, P. Perez and F. Heitz. "Discrete Markov modeling and inference on the quad-tree." IEEE Trans. Image Processing, 390-404, 2000.

[15] A. Voisin, V. Krylov, G. Moser, J. Zerubia and S.B. Serpico. "Supervised Classification of Multi-sensor and Multi-resolution Remote Sensing Images with a Hierarchical Copula-based Approach." IEEE Trans. on Geoscience and Remote Sensing, 33463358, 2014.
[16] Annabelle Chardin. Modeles energetiques hierarchiques pour la resolution des problemes inverses en analyse d'images application a la teledetection. $\mathrm{PhD}$ thesis, Rennes 1, 2000.

[17] S. Martinis, A. Twele, S. Voigt, "Unsupervised Extraction of Flood-Induced Backscatter Changes in SAR Data Using Markov Image Modeling on Irregular Graphs," IEEE Transactions on Geoscience and Remote Sensing, vol.49, no.1, pp.251-263, 2011.

[18] P.A. Devijver, "Image segmentation using causal Markov random field models", In Pattern Recognition, pp. 131-143. Springer Berlin Heidelberg, 1988.

[19] J. Whittaker. Graphical models in applied multivariate statistics, Wiley Publishing, 2009.

[20] Q.R. Razlighi, N. Kehtarnavaz, A. Nosratinia, "Computation of Image Spatial Entropy Using Quadrilateral Markov Random Field," IEEE Transactions on Image Processing, vol.18, no.12, pp. 2629$2639,2009$.

[21] S. Yousefi, N. Kehtarnavaz, C. Yan, "Computationally Tractable Stochastic Image Modeling Based on Symmetric Markov Mesh Random Fields," IEEE Transactions on Image Processing, vol.22, no.6, pp.2192-2206, 2013.

[22] S. Yousefi and N. Kehtarnavaz, "Generating symmetric causal Markov random fields,” IEEE. Lett., vol. 47, no. 22, pp. 1224-1225, 2011.

[23] A.C. Bovik. Handbook of image and video processing. Academic press, 2010.

[24] Pérez Modèles et algorithmes probabilistes pour l'analyse d'images, HDR, Rennes univ., 2003.

[25] C.M. Bishop. Pattern Recognition and Machine Learning, Information Science and Statistics. 2006

[26] C. Bouman and M. Shapiro, "A multiscale random field model for Bayesian image segmentation," IEEE Trans. Image Process., vol. 3, no. 2, pp. 162-177, 1994.

[27] I. Hedhli, G. Moser, S.B. Serpico, J. Zerubia, "New hierarchical joint classification method for SAR-optical multiresolution remote sensing data," EUSIPCO, pp.759-763, 2015

[28] I. Hedhli, G. Moser, J. Zerubia, S.B. Serpico, "New cascade model for hierarchical joint classification of multitemporal, multiresolution and multisensor remote sensing data," ICIP pp.5247-5251, 2014 